\title{
The Teaching of Geometric (Isometric) Transformations at Secondary School Level: What Approach to Use and Why?
}

\author{
Samuel Mashingaidze ${ }^{1}$ \\ ${ }^{1}$ Department of Applied Education, Faculty of Education, Midlands State University, Gweru, Zimbabwe \\ Correspondence: Samuel Mashingaidze, Department of Applied Education, Faculty of Education, Midlands State \\ University, P. Bag 9055, Gweru, Zimbabwe. E-mail: mashingaidzess@msu.ac.zw
}

Received: February 24, 2012 Accepted: July 30, 2012 Online Published: November 30, 2012

doi:10.5539/ass.v8n15p197 URL: http://dx.doi.org/10.5539/ass.v8n15p197

\begin{abstract}
The topic of Geometric transformation is topic number 10 out of 11 topics on the national ordinary level mathematics syllabus. It involves both analytic and algebraic geometry. Analytic because it can be approached using the graphical perceptive, and algebraic because matrix theory can be applied. It requires learners to have a good grasp of a number of skills, the so called assumed knowledge. Thus Teachers of mathematics before designing, selecting and implementing a lesson, ought to understand the knowledge that their students already have (or do not have). This is because one mathematical topic depends on another, early strengths support later progress while earlier weaknesses compound into greater debility. Such information is extremely useful for planning instruction. Assumed knowledge base covers topics such as vectors, construction of shapes, symmetry, properties of shapes, Cartesian equations and graphs, similarity and congruency, etc. Because of such demands learners are found wanting especially where it involves deciding the type of transformation. It is thus the thrust of this paper to approach transformations first by using graphs. Such an approach may cause understanding and enjoyment amongst teachers and learners in the topic. This approach aims at exposing the student to real practical experiences with transformations. Brief synopses on issues of the subject content are provided.
\end{abstract}

Keywords: translation, reflection, rotation

\section{Introduction}

Transformations are somewhat a difficulty topic of the ordinary level mathematics syllabus. It usually comes barely towards the end of the syllabus, and as such it is either skipped or hurriedly done by most teachers of mathematics. Students and teachers, both, exhibit serious shortcomings in their understanding of transformations. During a workshop which the author conducted with mathematics teachers in Mberengwa district, it emerged that Transformation as a topic gives teachers the least pleasure, yet it is considered important in supporting students' development of geometric and spatial thinking (Hollerbrands, 2008). In this workshop teachers voiced mixed feelings about how they handle the teaching of transformations. Some said they never attempt to teach it because they don't quite understand it, whilst others said they usually teach it when there is very little time left to finish the syllabus resulting in a crush programme by teachers. However studies have revealed evidence suggesting that teaching geometric transformations is feasible and may have positive effects on students' learning of mathematics (Edwards, 1989).

With transformations, it appears problems encountered by the students are a result of lack of conceptual understanding and might also be a result of the teaching they experience in learning transformations. Nziramasanga commission (1999) lamented the poor state of mathematics instruction in Zimbabwe and averred that the problems of quality of mathematics instruction and learning are from diverse sources. The mathematics teacher, however, has fallen victim by being accused to be responsible for the low quality of student performance in our secondary schools (Curriculum Team Research report, 2010). Foster (2007) highlights that if students are taught abstract ideas without meaning, this might not develop their understanding. Teachers appear to have difficulties with their own content knowledge. If a concept becomes more sophisticated for the teacher, it frequently becomes a barrier to students' understanding. The O-level text books also fail to present the content in such an elaborate way that could provide sufficient room for students to develop relational knowledge (Nziramasanga commission, 1999).

Against such a background it has to be acknowledged that the article comes at an appropriate time, and that there 
is always much interest amongst the general public about standards of achievement in mathematics. Mathematics occupies a core status in the secondary school curriculum because it is a key to opening career opportunities for students (Brumbaugh \& Rock, 2001).Nevertheless this subject warrants an empirical study to answer a number of questions, since there are several suggestions that there is limited research on transformational geometry (Boulter \& Kirby, 1994, Hollerbrands, 2003).

\section{Teaching Transformations}

Transformation can be divided into two areas, isometries and non-isometries. Isometries include Translation, Reflection and Rotation. Whereas non-isometries include enlargement, Shear and Stretch. This article focuses on the teaching of isometries.

According to Greer (1983:217) a Transformation, "describes the relation between a point and its image". In other words, "a transformation is a geometrical operation which transforms or maps a set of points onto another set (the image)" (Talbert,? :124). The word transforms means "changes". In geometry a transformation changes the position of a shape on a coordinate system. That is if a point $P$ has been transformed into a point $P^{\prime}$ (read as $P$ prtme), then $P$ is called the pre-image or object and the point $P$ is called the image. Transformations which preserve the shape and size of the original geometrical figure are called isometries (Talbert, ?). In this case the image has the same dimensions as the original given shape, as shown in the example in Figure 1 below.

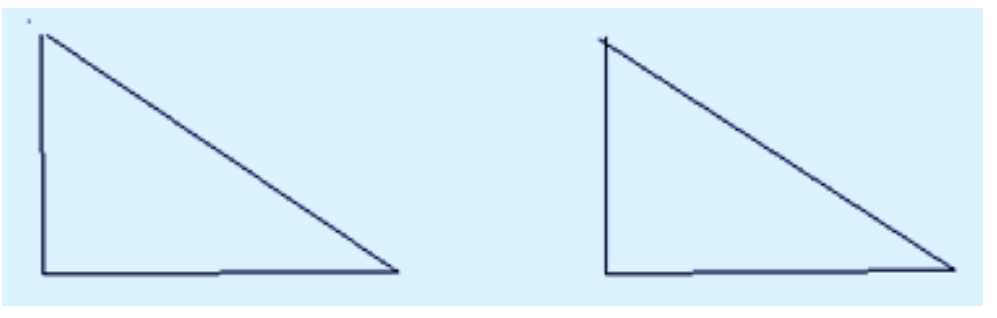

Figure 1.

The effective teaching of transformation, just like any topic in mathematics, must include not only facts to be mastered but also an appropriate and logical system of cognitive activity. In other words, what is required is for the student to acquire relational understanding more than procedural understanding. "Getting to know mathematics does involve much concrete experience and grounding in its central concept like number, shape and size comes through interaction with real objects and activities involving them", (Bently and Malvern, 1983). Learning mathematics is first and foremost learning how to do mathematics. The prime concern of mathematics teaching is to enable pupils to develop skills and to use them. That is, at the end of the day what is important is what the pupil can do, not what we have taught. Instruction should be developed from problem situations as long as the situations are familiar, conceptions are created from objects, events and relationships in which operations and strategies are well understood, (NCTM, 1989). When teaching, teachers must also be concerned with developing the pupil's mind so that one can solve problems for oneself.

The provision of pictures and diagrams, or the lack of them, can make sharp differences to the difficulty of a mathematics topic. Transformation problems can be solved by any of the two methods, that is, graphically and algebraically. And assessment of learning must include providing opportunities for students to choose how they solve a problem and then observing how they do it as well as seeing if they get it right or wrong (Bently and Malvern, 1983). The implication of all this is that in establishing important ideas in the mind of the pupil we have to recognise that the same concept applied in different cases becomes more difficult or less difficult depending on the situation.

As with Transformations, the idea, is to start with a much more practical approach so that pupils can visualize and they should do many such problems until they have a firm grasp of what they are doing when performing a transformation. Analytic Geometry, a great invention of Descartes and Fermat, is a branch of algebra that is used to model geometric objects - points, (straight) lines, and circles being the most basic of these (Brannan et al, 2002). In the algebraic approach, the main focus of teaching is to explain to the learners a way to determine the relationship between the object and its image in the form of an equation. Hawking (1999) believes that equations are the boring part of mathematics.

As when harder problems are introduced students must be made to appreciate that the procedure they have acquired is based on simple but sound mathematical principles. Although a specific idea might be forgotten the context in which it is learned can be remembered and the idea re-created. In this way students develop a framework of support that can be drawn upon in the future when rules may well have been forgotten but the structure of the 
situation remains embedded in the memory as a foundation for reconstruction (NCTM, 2000). In this article the author proposes that the teaching of transformation that begins by employing the graphical/analytic approaches further enhances mathematics teaching and learning in the unit. Sometimes deciding on which method to use depends on how the question has been set.

\subsection{Geometry Concepts}

For any successful teaching and learning it is also important to establish whether students possess the correct assumed knowledge base. More often than not teachers simply assume students know what is required to study transformation when in some cases they don't. The following may constitute the pre-knowledge base;

1) Symmetry (For Reflection)

2) Geometric Constructions by ruler and compass only (For all transformations except translation)

3) Vectors (For Translation)

4) Cartesian systems (For all transformations)

5) Matrices (For all transformations)

6) Area (For all transformations)

7) plane shapes (For all transformations)

8), etc.

The following concepts, defined, can be derived from the above list of pre-knowledge base;

1) Geometry is an aspect of mathematics that deals with the study of different shapes. The shapes may be plane or solid. (Telima, 2011)

2) A matrix is a mathematical unit in which the elements are numbers arranged in rows and columns. In other words matrices are used to present numerical information in a compact form, e.g. $\left(\begin{array}{ccc}3 & 3 & 7 \\ 8 & 0 & 23\end{array}\right)$

3) A square matrix has the same number of rows as it has columns, e.g. $\left(\begin{array}{ll}2 & 0 \\ 2 & 2\end{array}\right)$ has 2 rows and 2 columns

4) A point in geometry defines a position in space and has no dimensions.

5) A line joins two points and its length is a measure of the distance between points. The shortest line between any two points is called a straight line, and all other lines are called curves.

6) A plane has two dimensions and is defined by two intersecting lines.

7) A plane shape is a geometrical form such that the straight line that joins any two points on it wholly lies on the surface. (Telima, 2011)

8) Two shapes are said to be congruent when they are exactly the same size as well as the same shape. The symbol for congruent is $\Xi$, which means equal in all respect.

9) A line of symmetry is a mirror line that divides a plane figure into two identical halves, each being a mirror image of the other.

10) Locus is a line or curve joining all points in the plane which satisfy the given conditions. Constructions referred to in this article are performed with a ruler and a pair of compasses only.

11) Area defines the quantity of the plane surface bounded by the sides of the polygon. It has two dimensions (e.g. length and breadth) and is measured in square units.

12) Cartesian plane is made up of two axes, the $\mathrm{x}$-axis drawn parallel to the horizontal or top of the page and the y-axis drawn vertically. Each point in the plane of the page can be represented by an ordered pair of values $(x ; y)$ called Cartesian coordinates of the point.

13) A vector is a quantity possessing both magnitude and direction, e.g. atsplacement.

[Adapted from Channon et al (1996)]

All of the above constitute pre-requisite skills to the successful study of the topic of Transformations. Perhaps this explains why such a topic would come right towards the end of the syllabus, after which most of the topics have been taught. Usually resources become one of the most striking challenges facing the student. Resources required for a successful journey in this topic include; 
1) Ruler and compass. Ideally a mathematical set.

2) Sharp Pencil and rubber.

3) Graph book.

4) Time for practice.

\section{Exploring and Drawing with Transformations}

\subsection{Translation}

A translation is a movement in a straight line, vertical, horizontal or inclined (Channon et al, 1996). A translation can be in any direction, vertical, horizontal or inclined. Fig 2 below shows a translation of the letter H. A translation is when the same displacement vector is added to the position vector of every point. In other words the plane shape is transformed by the displacement vector. Two methods can be used, graphical and algebraic. The former is more practical than the latter, it is more hands on. And hence it is more appropriate to start with the graphical method and conclude with the algebraic approach. The former allows the student to see the reason for learning. In a study carried out by Xenia \& Demetra (2009) it emerged that students perform better in translation tasks than the other types.

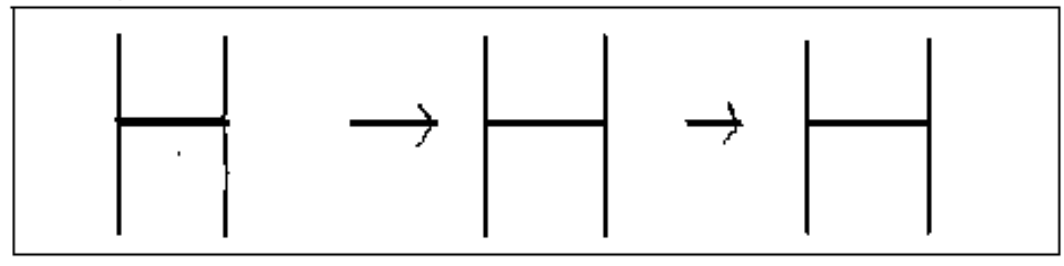

Figure 2.

\section{Graphical}

Let $\mathrm{U}$ be a vector quantity. In this case it denotes a movement of 7 units parallel to the $\mathrm{x}$-axis followed by 3 units parallel to the y-axis. , i.e. $U=\left(\begin{array}{l}7 \\ 3\end{array}\right)$

Since the movement of the object translates to every point of the object moving the same distance and in the same

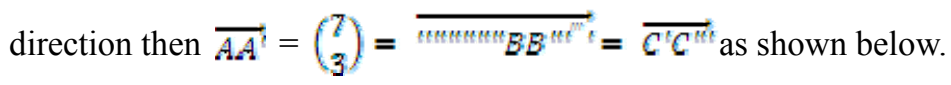

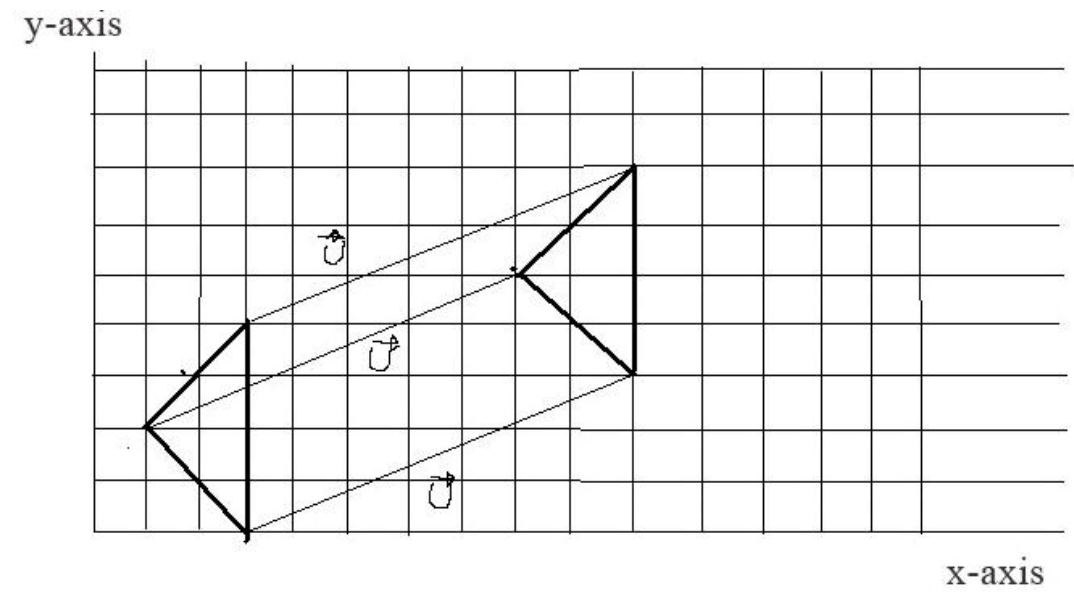

Figure 3.

\section{$\underline{\text { Algebraic }}$}

In Figure 3, $\mathrm{U}$ is a vector quantity with magnitude and direction. Thus to describe $\mathrm{U}$ requires the reader to recall the concept of a vector (Assumed knowledge). Are you well versed in this concept?

In this transformation every point of the object is mapped onto its image by an identical vector, $U$, called the 
translation vector/displacement vector.

This is can be written in the form of a transformation equation connecting the object to its image as shown below.

$$
\mathrm{O}+\mathrm{T}=\mathrm{I}
$$

Where $\mathrm{O}$ is the position vector of object point, $\mathrm{T}$ is the translation vector and, $\mathrm{I}$ is position vector of the corresponding image point.

\section{Activity 1.}

The vertices of a triangle are $A(3 ; 0), B(1 ; 2)$ and $C(3 ; 4)$. If TRIANGLE ABC is translated by vector $\left(\begin{array}{l}7 \\ 3\end{array}\right)$ find the coordinates of the vertices of its image.

\section{Solution:}

Let $\triangle \mathrm{A}^{\prime} \mathrm{B}^{\prime} \mathrm{C}^{\prime}$ be the image of $\triangle \mathrm{ABC}$ under the translation.

If $A(3 ; 0)$ then position vector $\overrightarrow{O A}=\left(\begin{array}{l}3 \\ 0\end{array}\right)$,

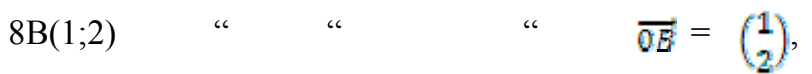

$$
\begin{aligned}
& 8 \mathrm{C}(3 ; 4) \quad \text { * } \quad \text { " } \quad \overrightarrow{O C}=\left(\begin{array}{l}
3 \\
4
\end{array}\right) \text { [Assumed knowledge- vectors] }
\end{aligned}
$$

Using $\mathrm{O}+\mathrm{T}=\mathrm{I}$ for all the three points;

$$
\begin{aligned}
& \mathrm{A}(3 ; 0):\left(\begin{array}{l}
3 \\
0
\end{array}\right)+\left(\begin{array}{l}
7 \\
3
\end{array}\right)=\left(\begin{array}{c}
10 \\
3
\end{array}\right) \text { t.e } A^{\prime \prime \prime \prime}(10 ; 3) \\
& \mathrm{B}(1 ; 2):\left(\begin{array}{l}
1 \\
2
\end{array}\right)+\left(\begin{array}{l}
7 \\
3
\end{array}\right)=\left(\begin{array}{l}
8 \\
5
\end{array}\right) \text { t. e. } B^{\prime \prime m m}(8 ; 5) \\
& \mathrm{C}(3 ; 4):\left(\begin{array}{l}
3 \\
4
\end{array}\right)+\left(\begin{array}{l}
7 \\
3
\end{array}\right)=\left(\begin{array}{c}
10 \\
7
\end{array}\right) \text { t. e. } C^{\prime}(10 ; 7)
\end{aligned}
$$

In general if a point $\mathrm{P}(\mathrm{x} ; \mathrm{y})$ is translated to $\mathrm{P}^{\prime}\left(\mathrm{x}^{\prime} ; \mathrm{y}^{\prime}\right)$ by a translation vector $\left(\begin{array}{l}a \\ b\end{array}\right)$ then $\mathrm{P}^{\prime}\left(\mathrm{x}^{\prime} ; \mathrm{y}^{\prime}\right)$ is called the image of $\mathrm{P}(\mathrm{x} ; \mathrm{y})$ under the translation, and $\left(\begin{array}{l}x \\ y\end{array}\right)+\left(\begin{array}{l}\boldsymbol{a} \\ b\end{array}\right)=\left(\begin{array}{l}x^{\prime} \\ y^{\prime}\end{array}\right)$

\subsection{Reflection}

A reflection is the image you see when you look in a mirror (Channon et al, 1996). If you stand before a mirror you see exactly yourself (your image, same distance from the mirror as you are). If you walk towards the mirror your image simultaneously walks towards you or the mirror. In fig 4 , the symbol $\Lambda$ is the object and is its image under a reflection in the mirror line as shown.

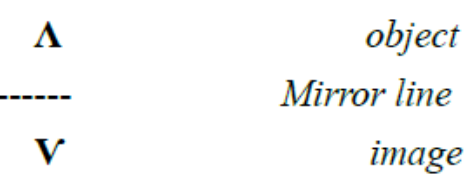

Figure 4.

\section{Graphical}

In this approach we explore closely the relationship between the object and its image. In fig 5, below, $M$ is the object and $M^{\prime}$ is the image.

The fig below shows a reflection of object $M$ in the line $L$ producing the image $M^{\prime}$. 


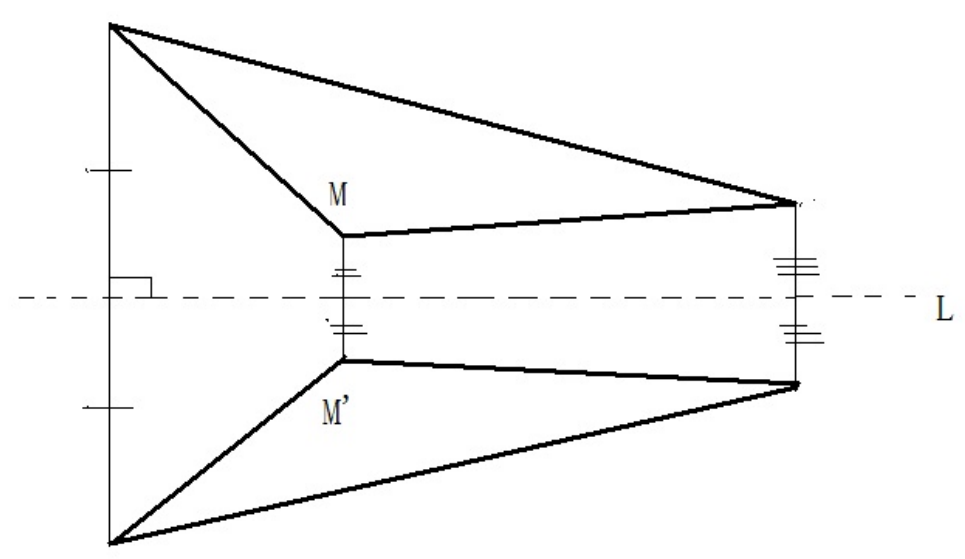

Figure 5.

Such an analysis brings out the following important observations;

A reflection is a transformation of a plane having the following properties;

- The line joining the object and corresponding image is perpendicular to the mirror line, $\mathrm{L}$ (which is a perpendicular bisector of the line joining any two corresponding points)

- Any point on the reflected object is the same distance as its corresponding object point from the line of reflection.

- All points on the mirror line are unchanged or are not affected by the transformation (invariant points).

- The image and the object are oppositely congruent to each other.

\section{Activity 2.}

Draw the image of the TRIANGLE, given below, under a reflection about the line $y=4$

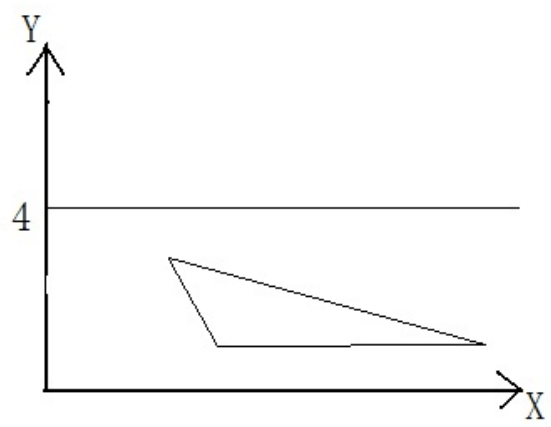

Figure 6.

\section{Solution:}

In this task you are required to demonstrate your skills in using mathematical apparatus the ruler, set square and compass/divider as demonstrated below.

\section{Stage 1.}

Using ruler and set square draw straight lines from each point of the object ensuring that they cross and are perpendicular to the mirror line $(\mathrm{y}=4)$ as shown below. 


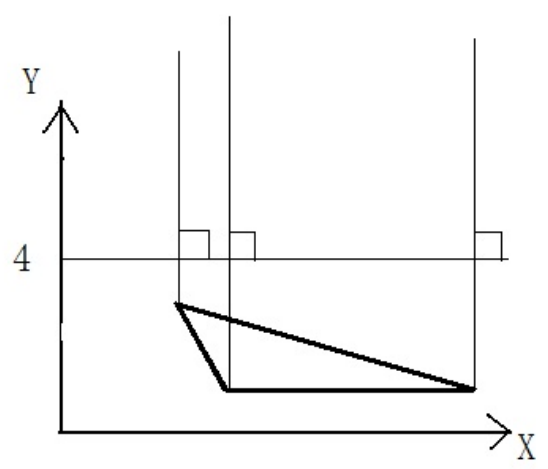

Figure 7.

\section{Stage 2.}

Using a divider /pair of compasses, measure out the distance of each object point from the line of reflection and along the perpendicular lines drawn in stage 1 (and using the same opening of the divider/pair of compasses) mark a point on the other side of the mirror line as shown below.

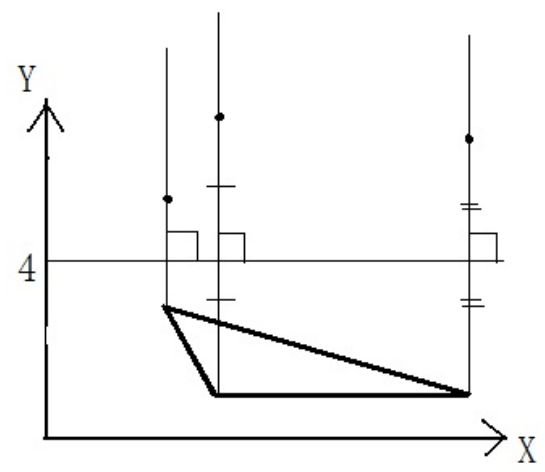

Figure 8.

Stage 3 .

Join the points to form the image of the triangle under this transformation, as shown below.

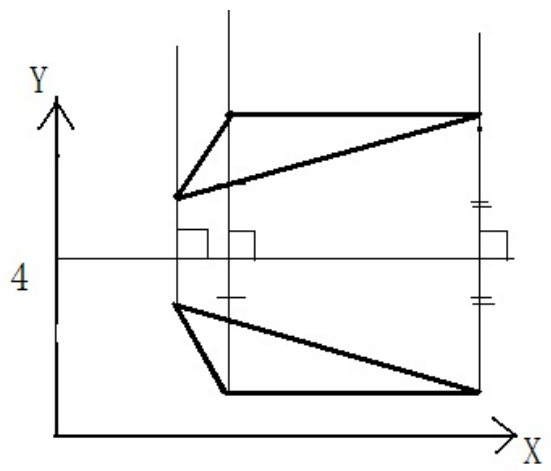

Figure 9 .

\section{$\underline{\text { Algebraic }}$}

Reflection in the Coordinate Axes

In this method a matrix for a transformation, called a matrix operator must be obtained. To find the matrix of the transformation, first sketch a unit square $\mathrm{ABCD}$ on the $\mathrm{x}-\mathrm{y}$ plane, as shown in fig 6 shown below.

(a) Reflection in the $x$-axis $(y=0)$

A quick way to find the matrix operator for this transformation is to use the following rule; Consider the identity 
matrix, $\mathbf{I}=\left(\begin{array}{ll}1 & 0 \\ 0 & 1\end{array}\right)$. We notice $\left(\begin{array}{l}1 \\ 0\end{array}\right)=\overrightarrow{O A}$ and $\left(\begin{array}{l}0 \\ 1\end{array}\right)=\overrightarrow{O C}$.

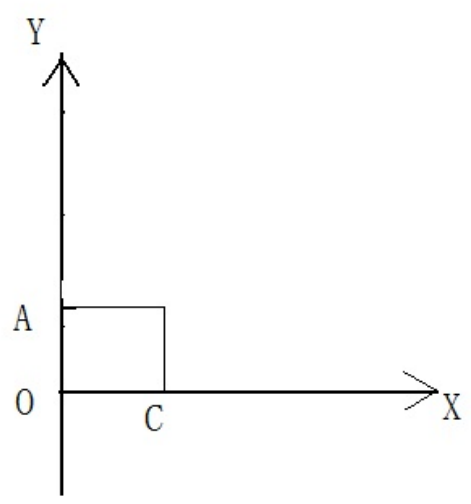

Figure 10.

Thus considering the images for $\mathrm{A}$ and $\mathrm{C}$ under a reflection about the $\mathrm{x}$-axis, that is, $\mathrm{A}^{\prime}(0 ;-1)$ and $\mathrm{C}^{\prime}(1 ; 0)$ we observe that $\left(\begin{array}{ll}1 & 0 \\ 0 & 1\end{array}\right) \rightarrow\left(\begin{array}{cc}1 & 0 \\ 0 & -1\end{array}\right),($ Bicknell, 1984).

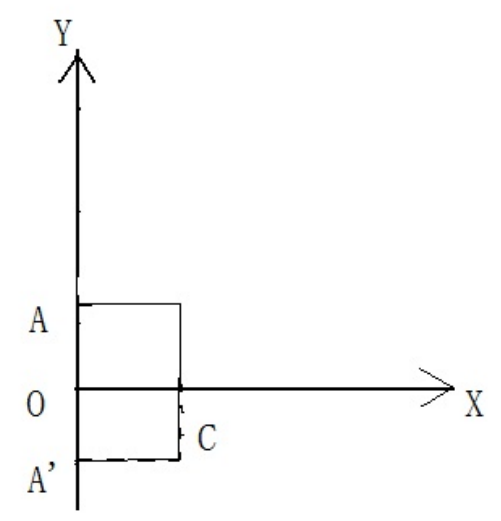

Figure 11.

NB: point $\mathrm{O}$ and $\mathrm{C}$ are not moved under this transformation and this is because they lie on the invariant line, the mirror line of reflection.

Thus $\left(\begin{array}{cc}1 & 0 \\ 0 & -1\end{array}\right)$ is called the matrix operator under a reflection about the x-axis. For any point $\mathrm{P}(\mathrm{x} ; \mathrm{y})$ in the plane, $\mathrm{M}\left(\begin{array}{l}x \\ y\end{array}\right)=\left(\begin{array}{cc}1 & 0 \\ 0 & -1\end{array}\right)\left(\begin{array}{l}x \\ y\end{array}\right)=\left(\begin{array}{c}x \\ -y\end{array}\right)$ where $\mathrm{M}$ is the matrix operator.

NB. The following becomes an equation that connects the object points to the corresponding image points.

$$
\mathrm{M} . \mathrm{O}=\mathrm{I}
$$

Where $\mathrm{M}$ is the matrix operator, $\mathrm{O}$ is object matrix, and $\mathrm{I}$ is the image matrix.

\section{(b) Reflection in the $y$-axis $(x=0)$}

The matrix operator can be obtained in a more or less similar procedure as above with the only difference being that we are reflecting about the y-axis. This is left an exercise for you. 
$\underline{\text { Task }}$

Prove that $\left(\begin{array}{cc}-1 & 0 \\ v & 1\end{array}\right)$ is the matrix operator under a reflection about the y-axis.

\section{Activity 3.}

The vertices of a triangle are $A(1 ; 2), B(2 ; 3)$ and $C(3 ;-4)$. If $\triangle A B C$ is reflected about the $x$-axis find the coordinates of the vertices of its image.

\section{Solution:}

In this question there is no need to draw the triangles. Knowledge skills required here are in matrix multiplication and vectors.

Position vectors of the object's points can be represented collectively as matrix $\mathrm{O}$, where

$\mathrm{O}=\left(\begin{array}{ccc}1 & 2 & 3 \\ 2 & 3 & -4\end{array}\right)$

Thus using $M . O=I$ we have $\left(\begin{array}{cc}-1 & 0 \\ 0 & 1\end{array}\right)\left(\begin{array}{ccc}1 & 2 & 3 \\ 2 & 3 & -4\end{array}\right)=\left(\begin{array}{ccc}-1 & -2 & -3 \\ 2 & 3 & -4\end{array}\right)$.

Resulting in the following image points; $\mathrm{A}^{\prime}(-1 ; 2), \mathrm{B}^{\prime}(-2 ; 3)$ and $\mathrm{C}^{\prime}(-3 ;-4)$.

Note that the $\mathrm{x}$-coordinate remains unchanged and the $\mathrm{y}$-coordinate only changes sign under this transformation, for example $(2 ; 6) \rightarrow(2 ;-6)$. In general $(a ; b) \rightarrow(a ;-b)$, whereas in the case of a Reflection in the $y$-axis $(x=0)$ the $y$-coordinate remains unchanged and the $x$-coordinate changes sign.

Thus in general $(\mathrm{x} ; \mathrm{y}) \rightarrow(-\mathrm{x} ; \mathrm{y})$.

Table 1. Summary of reflection, object $(a ; b)$

1) Find by a graphical method the equation of the mirror line for transformation

$(3 ; 5) \rightarrow(5 ; 3)$.

2) Reflect the word GAME (i) across the page (ii) down the page

(i)

\begin{tabular}{|l|l|}
\hline G & \\
\hline $\mathbf{A}$ & \\
\hline $\mathbf{M}$ & \\
\hline $\mathbf{E}$ & \\
\hline
\end{tabular}

(ii)

\begin{tabular}{|l|l|l|l|}
\hline $\mathbf{G}$ & $\mathbf{A}$ & $\mathbf{M}$ & $\mathbf{E}$ \\
\hline & & & \\
\hline
\end{tabular}




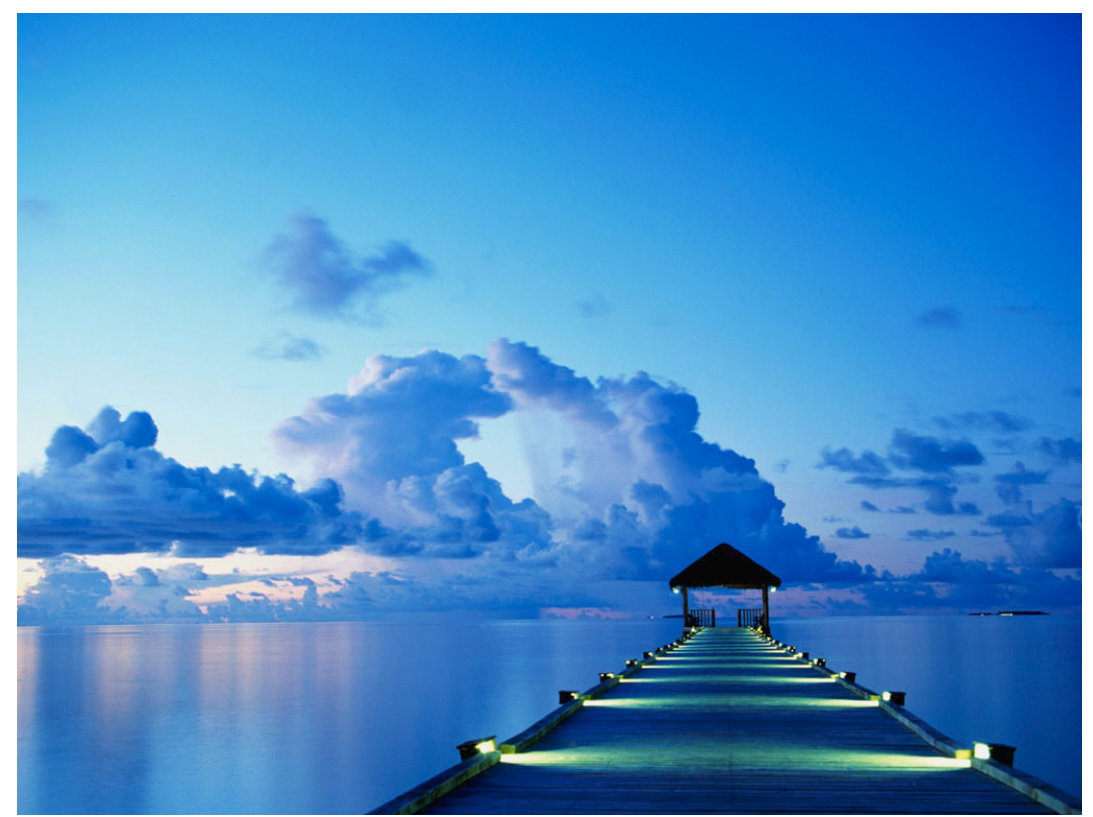

Figure 12. Reflections about the water level

\subsection{Rotation}

When a shape turns about a point, we say it rotates (Channon et al, 1996). A line joining any point to some fixed point if rotated about the fixed point defines a transformation called rotation as shown in fig 9 below.

A paired sample t-test for comparing students' mean performance in reflection tasks and in rotation tasks revealed that the mean difference is statistically significant in favour of reflections(Xenia \& Demetra, 2009). In other words, rotation tasks are more difficulty than reflection tasks. However spatial imagery cognitive style can significantly improve performance in rotation tasks (Xenia \& Demetra, 2009). This means that pupils must be exposed to the analytic approach more often than the algebraic approach.

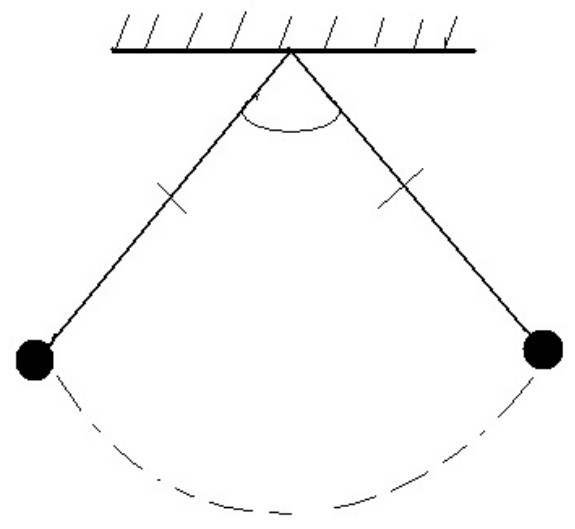

Figure 13. Swinging pendulum

With a swinging pendulum we observe that the object rotates about a fixed point through an angle. In this transformation the object maintains constant distance from the fixed point.

\section{Graphical}

When a shape/object turns or rotates, every point of it turns through the same angle. The distance from the centre of rotation is unchanged as shown in fig 9 .

\section{(a) Rotation about centres other than the origin}

Rotation about a centre other than the origin is usually solved more easily by graphically methods.

Given the image of the object the centre of rotation is found by the following method;

- Join each vertex to its image by a straight line 
- Construct perpendicular bisectors of the lines above.

- The centre of rotation is the meeting point of these perpendicular bisectors.

Apparatus used: ruler and pencil, protractor, compass/divider

\section{Activity 4.}

Find the coordinates of the image of the triangle below under a clockwise rotation through an angle of 90 degrees about the point $(4 ; 6)$.

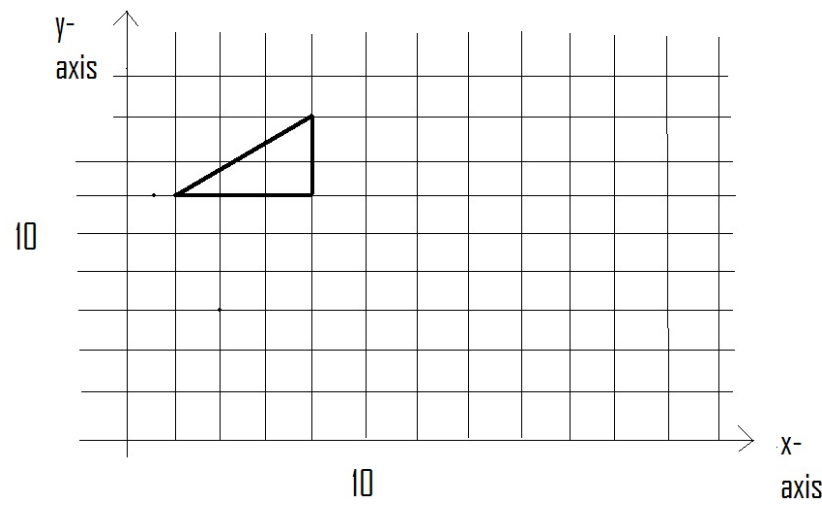

Figure 14 .

\section{Solution:}

Stage 1: Join each point of the object to the centre and use a protractor to measure out a clockwise 90 degree angle.

Stage 2: Using a compass/divider mark the image point same distance from the centre as its corresponding object point as shown below.

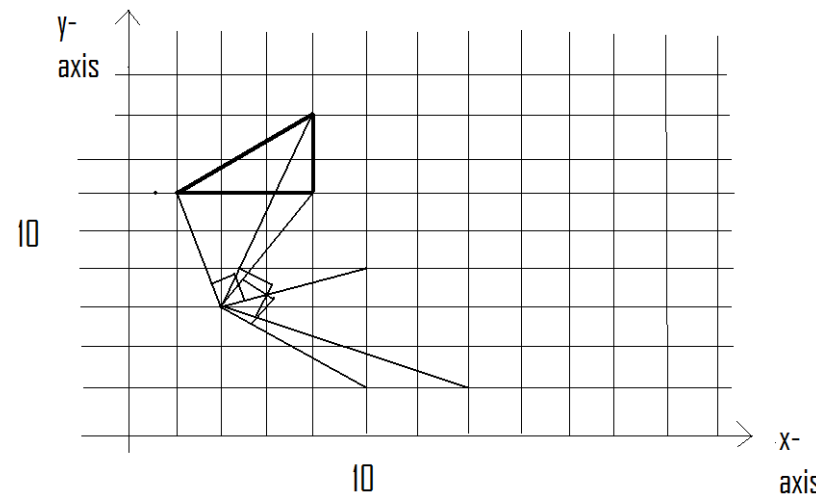

Figure 15.

Stage3: Join the three vertices of the image triangle.

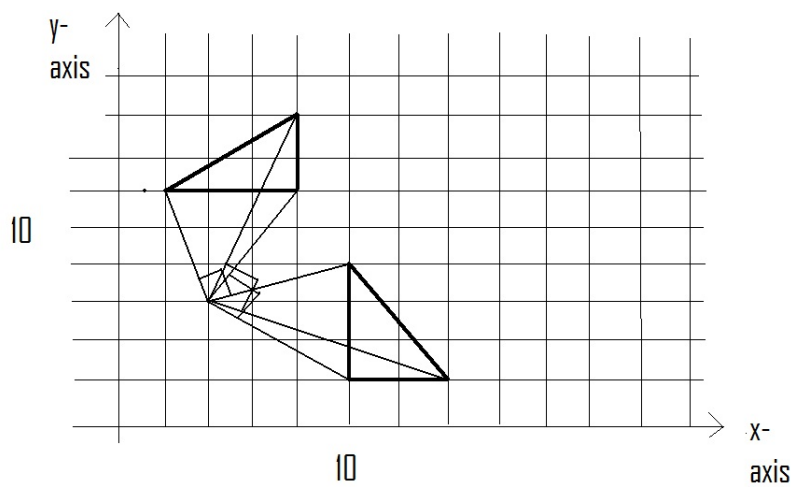

Figure 16. 


\section{What to Note:}

- Each point of the object has to rotate through 90 degrees clockwise regardless of its position.

- The distance of a point on the object/pre-image from the centre of rotation should be equal to the distance of its corresponding image point from the centre.

Algebraic

A rotation can be clockwise or anti-clockwise.

\section{(a) Rotation about the origin through 90' clockwise}

Stage 1: Sketch a square of unit length $\mathrm{OABC}$, as shown below.

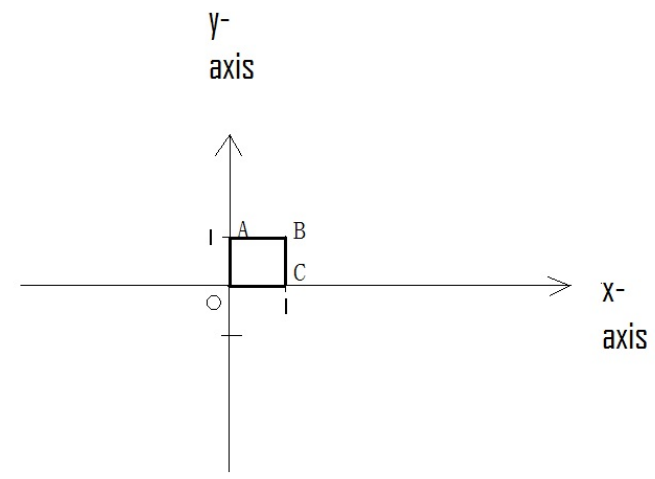

Figure 17.

Stage 2: Rotate the square about the origin through an angle of 90 degrees clockwise as shown below.

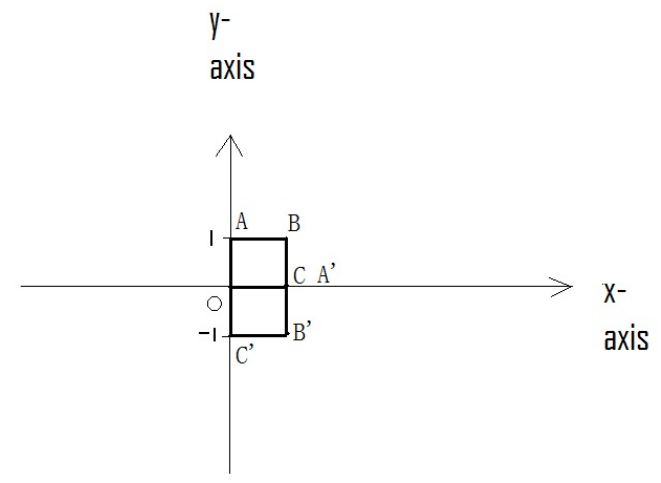

Figure 18.

A quick way to find the matrix operator for this transformation is to use the following rule; Considering the identity matrix, $\mathbf{I}=\left(\begin{array}{ll}1 & 0 \\ 0 & 1\end{array}\right)$, we notice $\left(\begin{array}{l}0 \\ 1\end{array}\right)=\overrightarrow{O A}$ and $\left(\begin{array}{l}1 \\ 0\end{array}\right)=\overrightarrow{O C}$.

Thus considering the images for $A$ and $C$ under a rotation about the origin, that is, $A^{\prime}(1 ; 0)$ and $C^{\prime}(0 ;-1)$ we observe that $\left(\begin{array}{ll}1 & 0 \\ 0 & 1\end{array}\right) \rightarrow\left(\begin{array}{cc}0 & 1 \\ -1 & 0\end{array}\right)$ (Bicknell, 1984)

Thus $\left(\begin{array}{cc}0 & 1 \\ -1 & 0\end{array}\right)$ is called the matrix operator of a rotation about the origin. For any point $\mathrm{P}(\mathrm{x} ; \mathrm{y})$ in the plane $\mathrm{M}\left(\begin{array}{l}x \\ y\end{array}\right)=\left(\begin{array}{cc}0 & 1 \\ -1 & 0\end{array}\right)\left(\begin{array}{l}x \\ y\end{array}\right)=\left(\begin{array}{c}y \\ -x\end{array}\right)$ where $\mathrm{M}$ is the matrix operator. 
Table 2. Summary of the different kinds of rotations about the origin--matrix operators can be obtained as in (a) above

\begin{tabular}{|c|c|c|c|c|c|}
\hline \# & Sense/direction & Angle in deg & Matrix & Equivalent & \\
\hline 1. & Clockwise & 90 & $\left(\begin{array}{c}0 \\
-1\end{array}\right.$ & $\begin{array}{l}\text { Ant-clockwise } \\
\text { deg }\end{array}$ & 270 \\
\hline 2. & Clockwise & 180 & $\left(\begin{array}{c}-1 \\
0\end{array}\right.$ & $\begin{array}{l}\text { Anti-clockwise } \\
\text { deg }\end{array}$ & 180 \\
\hline 3. & Clockwise & 270 & $\left(\begin{array}{l}0 \\
1\end{array}\right.$ & $\begin{array}{l}\text { Anti-clockwise } \\
\text { deg }\end{array}$ & 90 \\
\hline 4. & Clockwise & 360 & $\left(\begin{array}{ll}1 \\
0\end{array}\right.$ & $\begin{array}{l}\text { Anti-clockwise } \\
\text { deg }\end{array}$ & 360 \\
\hline
\end{tabular}

\section{Activity 5.}

The vertices of a triangle are $A(1 ; 2), B(2 ; 3)$ and $C(3 ;-4)$. If $\triangle A B C$ is rotated about the origin through 270 degrees clockwise,

(a) state the matrix operator for this transformation

(b) find the coordinates of the vertices of the image.

\section{Solution:}

(a) The matrix of the transformation is $\left(\begin{array}{cc}0 & -1 \\ 1 & 0\end{array}\right)$.

(b) Let $\triangle \mathrm{A}^{\prime} \mathrm{B}^{\prime} \mathrm{C}^{\prime}$ be the image of $\triangle \mathrm{ABC}$ under the rotation.

Using $M . O=I ;\left(\begin{array}{cc}0 & -1 \\ 1 & 0\end{array}\right)\left(\begin{array}{ccc}1 & 2 & 3 \\ 2 & 3 & -4\end{array}\right)=\left(\begin{array}{ccc}-2 & -3 & 4 \\ 1 & 2 & 3\end{array}\right)$

Therefore the image triangle has vertices $A^{\prime}(-2 ; 1), B^{\prime}(-3 ; 2)$ and $C^{\prime}(4 ; 3)$

\section{Full Description of a Transformation}

Table 3.

\begin{tabular}{lll}
\hline Transformation & What to describe \\
\hline 1. TRANSLATION & $\bullet$ & Name of transformation \\
2. REFLECTION & $\bullet$ & Displacement vector \\
3. ROTATION & $\bullet$ & Name of transformation \\
& $\bullet$ & Line of reflection/ Matrix of reflection \\
& $\bullet$ & Centre of rotation \\
& $\bullet$ & Angle and direction of rotation \\
\hline
\end{tabular}

\section{Summary}

Transformations are as important as any topic in mathematics. Transformations, for example, are an important stepping stone for the study of matrix theory. Thus students need to acquire skills in transformations for further application in mathematics. The direct algebraic approach that is used in our classrooms is one of the reasons learners believe that transformation is difficult to understand. It is important that the learners first understand it 
when try to visualise and draw. It has emerged in this write-up that the teaching of this topic should always commence with a practical approach - the graphical approach to consolidate understanding. Teachers ought to establish the knowledge that the students have (or do not have). Knowing in advance the difficulties that students may experience when learning new mathematical concepts and skills can help prepare teachers for the class. But getting desired objectives, teachers' content knowledge and content provided by textbooks also play a significant role for promoting students' relational knowledge and conceptual understanding of transformations regardless of their ultimate/future careers.

\section{References}

Bicknell, E. D. (1984). Project in secondary Mathematics: Book 5. Manzini: Macmillan Boleswa Publishers.

Boulter, D. R., \& Kirby. J. R. (1994). Identification of strategies used in solving transformational geometry problems. Journal of Educational Research, 87(5), 298-303. Retrieved from http://www.jstor.org/stable/27541933 http://dx.doi.org/10.1080/00220671.1994.9941257

Brannan, D. A., Esplen, M. F., \& Gray, J. J. (2002). Geometry. Cambridge University Press.

Brumbaugh, D. K., \& Rock, D. (2001). Teaching Secondary Mathematics. London: Lawrence Erlbaum Associates.

Channon, J. B., Smith, A. M., Head, H. C., Macrea, M. F., \& Chasakara, A. A. (1996). New general mathematics 3: An 'O'Level Course. Cape Town: CTP Printers.

Edwards, L. (1989). Children's learning in a computer microworld for transformation geometry (Doctoral dissertation, University of California, 1989). Dissertation Abstracts International, 51(6), 1943A.

Foster, D. (2007). Making meaning in Algebra examining students understandings and misconceptions. Assessing Mathematical Proficiency, 53, 163-176. Retrieved January 22, 2008, from http://www.msri.org/communications/books/Book53/contents.html http://dx.doi.org/10.1017/CBO9780511755378.017

Greer, A. (1983). A Concise CSE Mathematics. Cheltenham: Pitman press.

Hawking, S. (1999). Previous Quotes of the Day. Retrieved from http://www.skirtman.org

Hollebrands, K. (2003). High school students' understandings of geometric transformations in the context of a technological environment. Journal of Mathematical Behavior, 22(1), 55-72. http://dx.doi.org/10.1016/S0732-3123(03)00004-X

National Council of Teachers of Mathematics. (1989). Curriculum and evaluation standards for school mathematics. Reston, VA: NCTM.

National Education Advisory board. (2010). Curriculum team research report. Harare.

Nziramasanga. (1999). The Nziramasamga commission. Harare: Zimbabwe Government printers.

Talbert, J. F. (n.d.). Mathematics syllabus D. Harare: Longman.

Xenia, X., \& Demetra, P, P. (2009). Elementary Students' Transformational Geometry Abilities And Cognitive Style. Department of Education, University of Cyprus. 